\title{
La termoelectricidad: convirtiendo el calor en electricidad
}

\author{
Itzel de Jesús Soní Castro, Ana Bertha López Oyama y \\ Eugenio Rodríguez González
}

\section{Resumen}

Con el propósito de conocer una fuente renovable diferente a las ya conocidas hasta ahora, en este artículo, se presentan, de forma general, los mecanismos empleados en la generación de electricidad por medio de la termoelectricidad, la cual aprovecha el calor liberado por las máquinas o por algún proceso donde se realice un trabajo. Es importante conocer nuevas alternativas de generación eléctrica puesto que, cada día, millones de mexicanos nos conectamos a una red eléctrica, la cual se ha convertido en un aspecto fundamental para el desarrollo de la vida moderna. Asimismo, la electricidad nos sirve para proveer de limpieza, de seguridad, y de calor en la época invernal y para generar frío en los veranos calurosos. Además, es un eslabón crucial en las telecomunicaciones, en el transporte, en las actividades recreativas, entre otras. Actualmente, el mayor por ciento de la energía que se consume a nivel mundial proviene de combustibles fósiles, pero el uso desmedido de estas fuentes, para satisfacer la demanda creciente de electricidad, ha ocasionado un impacto negativo en el medio ambiente. Por tal motivo, en este artículo se busca que el lector conozca una alternativa para la generación de electricidad mediante el aprovechamiento del calor, una fuente inagotable y que, a su vez, disminuye el impacto negativo que se le produce en el medio ambiente.

Palabras clave: termoelectricidad, electricidad, medio ambiente.

\section{THERMOELECTRICITY: CONVERTING HEAT IN ELECTRICITY}

\begin{abstract}
With the purpose of knowing a renewable source, different from those already known so far, this article will present, in a general way, the mechanisms for generating electricity through thermoelectricity, which takes advantage of the heat released by machines or some processes that perform work. It is important to know new alternatives for electricity generation. Everyday millions of Mexicans connect to the electricity grid because it has become a fundamental aspect for the development of modern life, which provides safety, cleaning, heat in winter and cold in summers, in addition of being a crucial link in telecommunications, transportation, recreational activities, among others. Currently, the largest percent of the energy consumed worldwide comes from fossil fuels; since, the excessive use of these sources to satisfy the growing demand for electricity has caused a negative impact on the environment. For this reason, this article seeks for the reader to know an alternative for the generation of electricity using heat, which is an inexhaustible resource and that, in turn, reduces the negative impact on the environment.
\end{abstract}

Keywords: thermoelectricity, electricity, environment.

Recepción: 21/01/2021. Aprobación: 21/04/2021. http://doi.org/10.22201/cuaieed.16076079e.2021.22.4.9 
"La termoelectricidad: convirtiendo el calor en electricidad"

Itzel de Jesús Soní Castro, Ana Bertha López Oyama y

Eugenio Rodríguez González

Vol. 22, Núm. 4, julio-agosto 2021

Revista Digital Universitaria

Itzel de Jesus Soní Castro

itzel_soni@hotmail.com

Ingeniera química por el Instituto Tecnológico de Ciudad Madero en 2010, con una maestría en Tecnología Avanzada por el Instituto Politécnico Nacional, Centro de Investigación en Tecnología Avanzada (cicata Unidad Altamira) en 2015, actualmente cursa el doctorado en cicaTA Unidad Altamira.

\section{Ana Bertha López Oyama}

$$
\begin{array}{r}
\text { alopezoyama@hotmail.com } \\
\text { orcid.org/0000-0002-6317-2569 }
\end{array}
$$

Cursó estudios de doctorado en Ciencia de Materiales en la Universidad de Sonora. Actualmente, se encuentra adscrita al Centro de Investigación en Ciencia Aplicada y Tecnología Avanzada Unidad Altamira del Instituto Politécnico Nacional comisionada como catedrática CONACYT, donde desarrolla trabajos de investigación científica, formación de recursos humanos y divulgación de la ciencia.

\section{Eugenio Rodríguez González}

eugenior62@gmail.com

orcid.org/0000-0003-4038-3918

Licenciatura en Física y maestría en Ciencias en el área de Física Nuclear por la Universidad Técnica de Dresden (TUD), Alemania. Doctor en Ciencias por la Universidad Estadual de Campinas (UniCAMP), São Paulo, Brasil (2000-2004). Pos-Doctorado en el Instituto de Física "Gleb Wataghin", en la Universidad Estadual de Campinas, São Paulo, Brasil (2004-2009). Actualmente, está adscrito al Instituto Politécnico Nacional, Centro de Investigación en Ciencia Aplicada y Tecnología Avanzada (cICATA Unidad Altamira), Tamaulipas.

Fue profesor investigador en la Universidad de Oriente (UотE) Santiago de Cuba, Cuba (1987-1988) y, también en la Universidad de la Habana (UH) Ciudad Habana, Cuba (1988-2000). Asimismo, fue profesor colaborador en la Universidad Estadual de Campinas, São Paulo, Brasil (2004-2009). Actualmente, es profesor investigador en el Instituto Politécnico Nacional, cIcATA-Altamira, en Altamira Tamaulipas, desde 2009. Además, es miembro del Sistema Nacional de Investigadores, nivel ॥. Y desarrolla su formación en áreas como: óptica, optoelectrónica, nanotecnología, materiales fotovoltaicos y materiales termoeléctricos. 
"La termoelectricidad: convirtiendo el calor en electricidad"

Itzel de Jesús Soní Castro, Ana Bertha López Oyama y

Eugenio Rodríguez González

Vol. 22, Núm. 4, julio-agosto 2021

Revista Digital Universitaria

\section{Introducción}

Seguramente, en algún momento de tu vida has leído que: "la energía no se crea ni se destruye, solamente se transforma". A partir de este principio ponderal se establece que la energía se puede convertir de una forma a otra, y esto es de gran relevancia en el estudio de procesos físicos y químicos, ya que, tanto los seres humanos y como las actividades diarias que desarrollamos, involucran el empleo de la energía ya sea para alimentarnos, movernos, trabajar, reproducirnos y hasta para llevar a cabo los procesos propios del metabolismo, además, la energía pone en funcionamiento dispositivos, equipos y herramientas eléctricas. Por ejemplo, el movimiento de un automóvil se inicia porque los octanos, presentes en la gasolina, liberan energía como resultado de un proceso conocido como combustión; también, el oxígeno que respiramos es el resultado de una reacción química iniciada por la interacción de la luz solar con las plantas verdes.

El concepto energía proviene del vocablo griego energós que significa "fuerza de acción" y se relaciona con la capacidad de un cuerpo para realizar un trabajo. El aprovechamiento de la energía para producir electricidad ha sido de gran interés a lo largo de los años. Los seres humanos, en busca de disminuir el daño al planeta, han descubierto procedimientos y materiales útiles para tales transformaciones. Quizás el proceso más conocido es el de los paneles fotovoltaicos, los cuales aprovechan la luz solar para convertirla en energía eléctrica.

\section{¿Qué es el calor?}

El calor es la energía térmica que se transfiere entre dos cuerpos en contacto que se encuentran a diferentes temperaturas y su símbolo es la letra q o Q. Esta transferencia de energía se puede cuantificar como pérdida o ganancia y puede ser medida mediante unidades de energía como el joule (J). El calor está relacionado con la temperatura, la cual es una medida de la energía cinética ${ }^{1}$ de los átomos que forman parte del sistema. Si dos objetos, a diferente temperatura, entran en contacto el calor se transfiere del más caliente al más frío hasta alcanzar la misma temperatura o un equilibrio térmico, debido a las colisiones entre las moléculas.

Diariamente, realizamos actividades cotidianas donde el calor es el actor principal, por ejemplo, en los alimentos este factor está presente mientras tomamos el café caliente por las mañanas para protegemos del frío. El calor puede aprovecharse para obtener energía eléctrica mediante el uso de materiales con propiedades muy específicas conocidos como termoeléctricos. Esto significa que, el calor liberado al caminar, al hacer funcionar un automóvil o a partir de un proceso industrial, se puede aprovechar para obtener energía

${ }^{1}$ Es la energía que un objeto tiene debido a su movimiento. eléctrica. Si buscas aprender más acerca del calor y de la temperatura, accede a este enlace para ampliar tus conocimientos. 


\section{¿Qué es un material semiconductor?}

Existen 3 tipos de materiales de gran importancia en la conducción de energía eléctrica: Ios conductores, los aislantes y los semiconductores. Los conductores son buenos conductores de electricidad, y entre ellos se encuentra el cobre y la plata. Los aislantes son malos conductores de electricidad como, por ejemplo, el plástico o la madera; y por último, tenemos a los semiconductores que, en determinadas condiciones, pueden conducir la electricidad.

Para comprender cómo funcionan los semiconductores, primero hay que visualizar la organización interna de un átomo. En su núcleo se encuentran los nucleones (protones y neutrones). Los electrones orbitan alrededor del núcleo distribuidos en capas o niveles energéticos, al más externo de ellos se le conoce como nivel de valencia (ver figura 1), los electrones de este nivel son los que pueden participar en la formación de un enlace con los átomos vecinos. El material semiconductor más utilizado es el silicio (Si)² el cual presenta 4 electrones en su capa de valencia. En el video podrás visualizar la estructura atómica y profundizar en el entendimiento de la organización de un átomo.

Figura 1. Representación de un átomo proveniente del modelo atómico de Bohr. Adaptado de Zapata, 2020

Video . Introducción al átomo (KhanAcademyEspañol, 2014).

${ }^{2}$ Las letras entre paréntesis después del nombre del elemento químico es su símbolo dentro de la tabla periódica de los elementos.
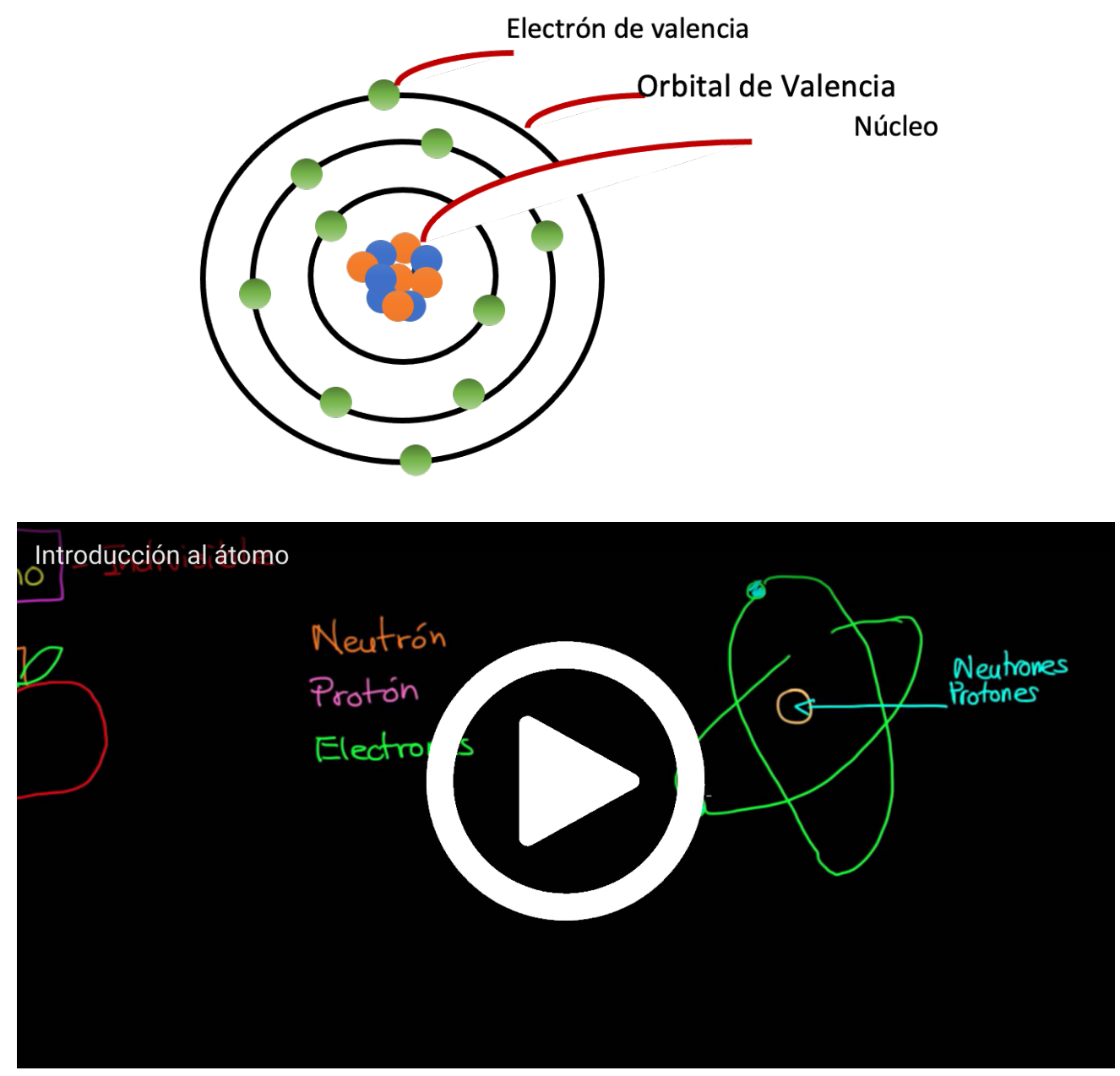
"La termoelectricidad: convirtiendo el calor en electricidad" Itzel de Jesús Soní Castro, Ana Bertha López Oyama y

Eugenio Rodríguez González

Vol. 22, Núm. 4, julio-agosto 2021

Revista Digital Universitaria

En un conglomerado de átomos (ej. silicio) estos van a interactuar entre ellos para formar enlaces. Los electrones de valencia se unirán a los de los átomos vecinos para formar un tipo de estructura conocida como cristal (ver figura 2a). A los niveles de energía en que se encuentran estos electrones de valencia se le llama la banda de valencia.

Sin embargo, un cristal puro no es un material útil electrónicamente, por lo cual se le introducen pequeñas cantidades de otros elementos para inducir la conductividad eléctrica. A este proceso se le conoce como dopaje y al elemento que se introduce se le denomina impureza o dopante. Los elementos químicos más utilizados como dopantes son el fósforo (P) y el boro (B), y cada uno de estos materiales presenta propiedades atómicas muy interesantes, el fósforo, por ejemplo, posee 5 electrones en su nivel de valencia, mientras que el boro posee 3.

Al dopar cristales de silicio con fósforo, solamente 4 electrones del fósforo se podrán enlazar a los 4 del silicio, en consecuencia, el quinto electrón del fósforo quedará en solitario y, por lo tanto, débilmente enlazado. Esa carga negativa puede donarse a otro átomo, y así, al material que posea cargas de este tipo se le conoce como semiconductor tipo-n (ver figura 2b). Si se le suministra energía al material, ese electrón puede desprenderse totalmente del átomo de fósforo y saltar a un nivel superior de energía, el cual le permite moverse libremente por el cristal. Al conjunto de niveles de energía que ocupan estos electrones, se le nombra como la banda de conducción.

Figura 2. Esquemas de (a) la estructura cristalina de silicio unidos por enlaces covalentes y (b) la estructura cristalina de silicio donde se le añade una impureza (fósforo, P) el cual presenta un electrón extra (electrón libre) aprovechable para la conducción eléctrica y (c) estructura cristalina de silicio donde se le añade una impureza (Boro, B) con valencia 3, presentando un déficit de electrón (hueco) aprovechable para la conducción eléctrica. Adaptado de Connor, 2019.

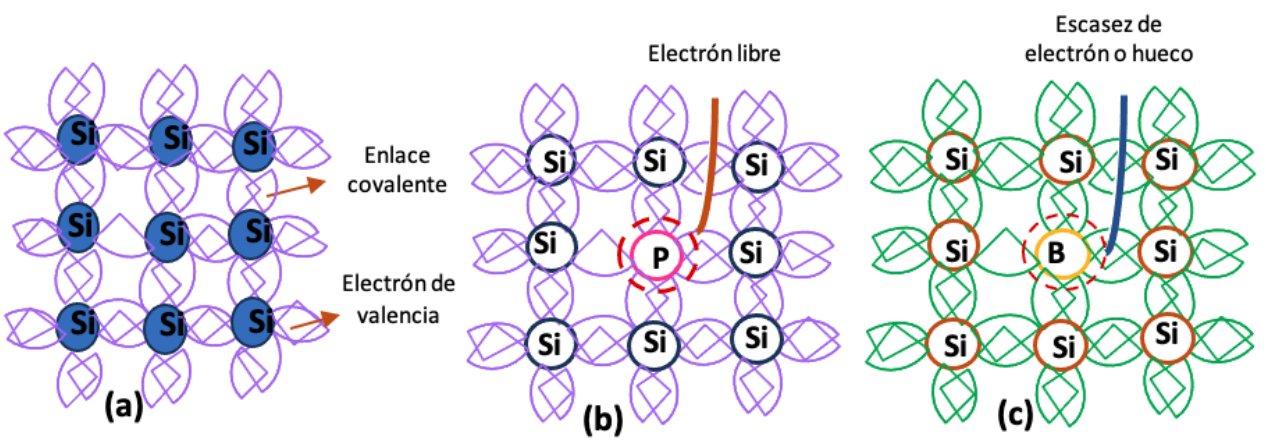

Por otra parte, si un cristal de silicio se dopa con boro, solamente 3 de los electrones de silicio pueden formar un enlace y el cuarto electrón del silicio se queda en solitario, formando un tipo de partícula conocida como hueco, la cual se comporta como una carga positiva y puede, fácilmente, aceptar un electrón. Un material con cargas de este tipo se le conoce como semiconductor tipo-p (ver figura 2c) y adquiere características de aceptor.

Si el proceso de dopaje se lleva a cabo de manera controlada, y si se introduce un elevado número de cargas, los cristales de semiconductores se pueden transformar en un material capaz de conducir la electricidad. A las cargas (electrones o huecos) introducidas en el material (huecos o electrones), mediante este proceso de dopaje, se les conoce como portadores de carga mayoritarios. 
"La termoelectricidad: convirtiendo el calor en electricidad"

Itzel de Jesús Soní Castro, Ana Bertha López Oyama y

Eugenio Rodríguez González

Vol. 22, Núm. 4, julio-agosto 2021

Revista Digital Universitaria

Recordemos que cargas iguales se repelen y que cargas contrarias se atraen. Como las cargas positivas (huecos) se sienten atraídas por las cargas negativas (electrones) originan un movimiento: el electrón se mueve a un hueco $y$, al hacerlo, deja una carga positiva en la posición anterior. Si se aplica energía a los semiconductores ya sea tipo-p o tipo- $n$, se induce un desplazamiento de cargas tal y como sucede en un conductor. La parte negativa del voltaje mueve a los electrones al lado positivo, lo cual resulta en un movimiento aleatorio de los electrones y los huecos, lo que da como resultado una corriente eléctrica. A la diferencia energética entre la banda de valencia y la banda de conducción, se le llama banda prohibida, debido a que no existen niveles intermedios para que el electrón pueda ocupar (ver figura 3).

Figura 3. Esquema de la teoría de bandas y representación del nivel de Fermi en semiconductores Adaptado de Connor, 2020

${ }^{3}$ Enrico Fermi fue un científico italiano conocido por el desarrollo del primer reactor nuclear y sus contribuciones al desarrollo de la teoría cuántica.

${ }^{4}$ Aplica, sobre todo, a partículas subatómicas, principalmente al electrón.

${ }^{5}$ Cada uno de los electrones, en un átomo con muchos electrones, ocupa una órbita caracterizada por números cuánticos (son 4 números cuánticos). El principio de exclusión de Pauli establece que no puede haber dos electrones con todos los números cuánticos iguales.

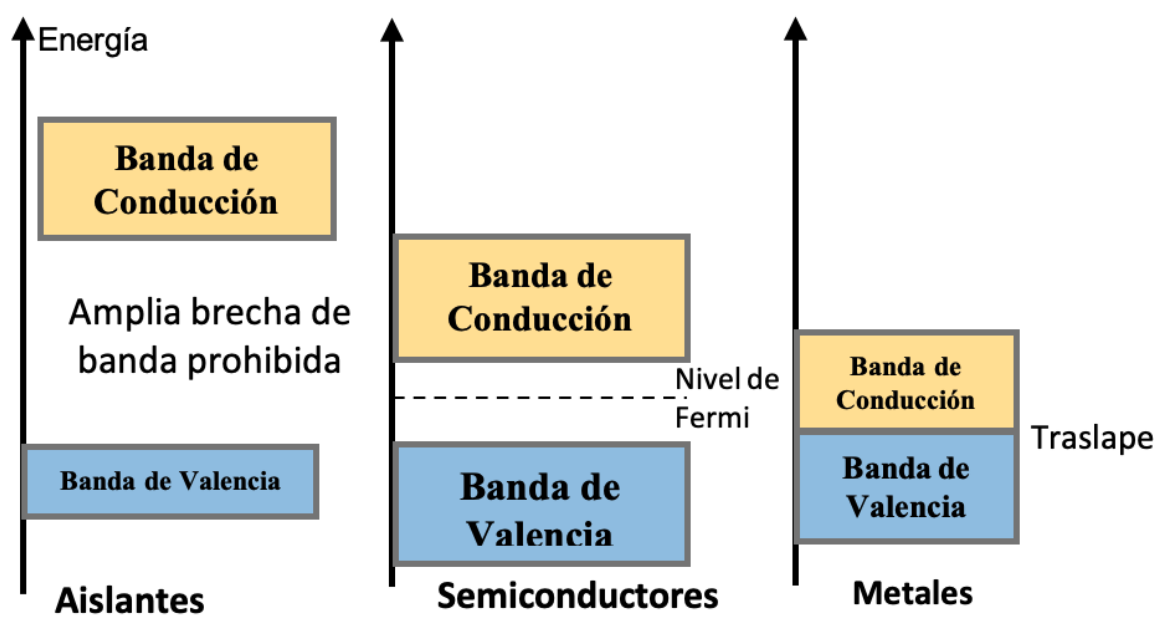

Un punto importante dentro de la banda prohibida es el nivel de Fermi ${ }^{3}\left(F_{L}\right)$. En los semiconductores, este nivel se encuentra en medio de la banda de valencia y la banda de conducción, mientras que, para los metales, se encuentra en la banda de conducción hipotética, que da paso a los electrones de conducción libre (Connor, 2020) (ver figura 3). El término $F_{\text {L }}$ es utilizado para explicar el conjunto más alto de niveles de energía de los electrones a la temperatura del cero absoluto, el cual proviene de las estadísticas de Fermi-Dirac ${ }^{4}$ donde se describe que, los fermiones o electrones no pueden existir en estados de energía idénticos de acuerdo con el principio de exclusión de Pauli ${ }^{5}$. Es importante el concepto del nivel de Fermi, ya que proporciona información sobre las velocidades de los electrones que participan en la conducción eléctrica (Olmo y Nave, 2020).

Los materiales semiconductores, tipo-n y tipo-p, resultan de gran interés para la fabricación de materiales termoeléctricos debido a la capacidad de formación de los portadores de carga aprovechables para la generación de corriente eléctrica por medio del efecto termoeléctrico. El comportamiento electrónico de los materiales semiconductores se caracteriza por la existencia y el movimiento de portadores de carga (electrones con carga negativa y "huecos" con carga positiva). 
La esta liga podrás encontrar más información sobre el funcionamiento de los dispositivos que emplean materiales semiconductores para su funcionamiento.

\section{¿Qué es el efecto termoeléctrico?}

Una vez que contamos con los materiales semiconductores, es necesario ponerlos a trabajar para que puedan convertir el calor en electricidad, o electricidad en calor a este fenómeno se le conoce como efecto Seebeck-Peltier o efecto termoeléctrico (Grupo de investigación de ingeniería térmica, s.f.).

El efecto Seebeck consiste en la capacidad de un cierto material de generar voltaje cuando se le suministra calor. Si se unen dos materiales semiconductores a través de un puente metálico (ver figura 4a) y se le aplica calor a esa unión, los portadores de carga mayoritarios de ambos materiales se desplazarán del extremo caliente al frío, lo cual generará una acumulación de cargas en el extremo frío de cada uno de ellos. Entre esos extremos se generará una diferencia de potencial o voltaje conocida como voltaje Seebeck o termovoltaje, cuya magnitud es proporcional a la diferencia de temperatura entre el extremo frío y caliente.

Por otro lado, si se aplica una diferencia de potencial entre los extremos libres de estos semiconductores (ver figura 4b), a través de ellos circulará una corriente. Y dependerá del sentido de esta, si los portadores de carga se desplazarán hacia los extremos libres de ambos materiales, enfriándola, o hacia la unión metálica, calentándola. A esto se le conoce como el efecto Peltier, a este par de materiales tipo $n$ y tipo $p$ unidos por un puente metálico (ver figura $4 a$ y 4b), se le denomina par termoeléctrico.

Figura 4. Representación esquemática del efecto Seebeck (a), efecto Peltier (b), y el de un dispositivo termoeléctrico (c). Adaptado de Vining, 2001.

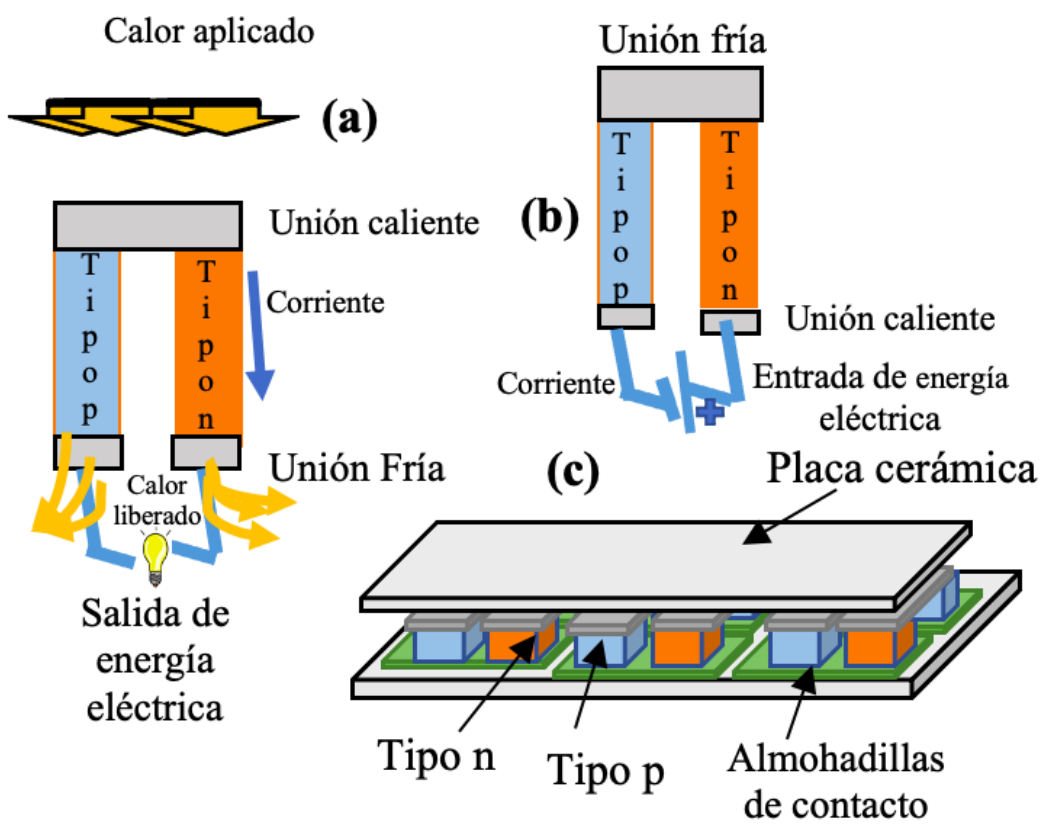




\section{Dispositivos termoeléctricos}

El efecto termoeléctrico se puede aprovechar para la generación de electricidad, medir temperatura, enfriar o calentar objetos. En la actualidad, se han desarrollado dispositivos termoeléctricos que permiten recuperar el calor disipado, en máquinas de combustión, para la generación de electricidad en sondas espaciales en el exterior del sistema solar, y también para la generación de sistemas de enfriamiento.

Para la fabricación de un dispositivo termoeléctrico, como una celda (ver figura 4c), deberán unirse centenas o miles de estos pares termoeléctricos, en serie y en paralelo para generar un voltaje y una corriente adecuada para que funcionen los dispositivos electro-electrónicos. La eficiencia de un material termoeléctrico se mide a través del factor de mérito (Z), definido como:

$$
Z=\frac{\sigma S^{2}}{k}
$$

El cual debe tener un alto coeficiente Seebeck(S), una elevada conductividad eléctrica $(\sigma)$ y una conductividad térmica $(k)$ lo más baja posible. Obtener un material con todas estas propiedades es un gran desafío tecnológico. Actualmente, la eficiencia de estos materiales se encuentra entre 1 y 2.2 lo que significa un rendimiento de conversión eléctrica entre 7\% y 15\%. Cabe señalar que, los materiales termoeléctricos, hasta ahora utilizados, son de naturaleza tóxica, de difícil procesamiento y de alto costo. Por tanto, la investigación científica se ha centrado en el entendimiento de nuevos materiales que tengan un mejor desempeño termoeléctrico y que presenten un bajo impacto al medio ambiente.

\section{Conclusión}

Desde los experimentos de Benjamín Franklin con el cometa en el año 1752 hasta la actualidad, el consumo de la electricidad ha crecido exponencialmente ya que, con el paso del tiempo se han encontrado varias maneras de mejorar nuestra vida a través de ella. El aprovechamiento de fuentes renovables o limpias aún tiene un largo camino por recorrer en términos de la eficiencia de conversión de energía. La dependencia de los combustibles fósiles y su impacto negativo en el medio ambiente motivan a la comunidad científica, a organizaciones no gubernamentales y a civiles a promover y a realizar actividades en pro del ambiente.

En nuestro país, se han establecido parques eólicos en Oaxaca, Baja California, Jalisco, Nuevo León, Tamaulipas y Chiapas (Grieta, s.f.) y fotovoltaicos en Chihuahua, Baja California Norte y Sur, Sinaloa, Nayarit, Jalisco, Ciudad de México, Querétaro, por mencionar algunos estados del país (Singh, 2019), para aprovechar las fuentes renovables de energía. El aprovechamiento del calor residual para convertirlo en electricidad ha generado interés en los grupos de investigación y en la iniciativa privada alrededor del planeta para desarrollar 
dispositivos termoeléctricos, ya que representan un campo benéfico para nuestro país y para el resto del mundo.

El uso del calor residual para la obtención de energía eléctrica es prometedor y pone en manifiesto la necesidad de desarrollar materiales en un laboratorio que cuenten con las características químicas requeridas para el proceso de transformación energética y, al mismo tiempo, que sea amigable con el medio ambiente.

\section{Referencias}

- Connor, N. (14 de diciembre de 2019). What is Doping of Semiconductors-Definition. Radiation Dosimetry. https://cutt.ly/2ngpbsk

* Connor, N. (9 de marzo de 2020). ¿Qué es el nivel de Fermi en semiconductores? Definición. Radiation Dosimetry. https://cutt.ly/angpc9G

* Grieta. (s.f.). Parques eólicos. https://www.grieta.org.mx/index.php/parqueseolicos/

* Grupo de Investigación de Ingeniería Térmica. (s.f.). Introducción a la Termoelectricidad. https://cutt.ly/angpxZr

* KhanAcademyEspañol. (2014, 7 de septiembre). Introducción al átomo [video]. YouTube. https://youtu.be/Jm9puMT16ro

Olmo M. y Nave R. (s.f.), El nivel de Fermi. HyperPhysics. https://cutt.ly/jngpj1F

* Singh, N. (2019, 9 de agosto). México alcanza los 50 parques fotovoltaicos operativos de gran escala: suman 4549 MW de potencia solar instalada. Energía Estratégica. https://cutt.ly/Zngpmid

Vining, C. B., (2001). Semiconductors are cool. Nature, 413, 577-578. https://doi. org/10.1038/35098159

- Zapata, F. (2020, 27 de julio). Modelo atómico de Bohr: características, postulados, limitaciones. Lifeder. https://www.lifeder.com/modelo-atomico-bohr/.

\section{Cómo CITAR ESTE ARTículo}

* Soní Castro, Itzel de Jesús, López Oyama, Ana Bertha y Rodríguez González, Eugenio. (2021, julio-agosto). La termoelectricidad: convirtiendo el calor en electricidad. Revista Digital Universitaria (RDU), 22(4). http://doi.org/10.22201/ cuaieed.16076079e.2021.22.4.9 